\title{
A PROPERTY OF POWER SERIES WITH POSITIVE COEFFICIENTS
}

\author{
P. ERDÖs, W. FELLER, AND H. POLLARD
}

The following theorem is suggested by a problem in the theory of probability. ${ }^{1}$

Let $p_{k}$ be a sequence of non-negative numbers for which $\sum_{0}^{\infty} p_{k}=1$, and let $m=\sum_{1}^{\infty} k p_{k} \leqq \infty$. Suppose further that

$$
P(x)=\sum_{0}^{\infty} p_{k} x^{k}
$$

is not a power series in $x^{t}$ for any integer $t>1$. Then $1-P(x)$ has no zeros in the circle $|x|<1$, and the series

$$
U(x)=\frac{1}{1-P(x)}=\sum_{0}^{\infty} u_{k} x^{k}
$$

has the property

$$
\lim _{n \rightarrow \infty} u_{n}=1 / m .
$$

(If $m=\infty$, we define $1 / m$ to be zero.)

We shall first give a proof in case $m<\infty$. The method used is not elementary, but yields somewhat more information than stated in the theorem. Later in this paper an elementary proof is given, valid for both $m<\infty$ and $m=\infty$.

We suppose that $m<\infty$. Let

$$
r_{n}=\sum_{k=n+1}^{\infty} p_{k}, \quad R(x)=\sum_{0}^{\infty} r_{n} x^{n} .
$$

Then $m=\sum_{0}^{\infty} r_{k}$ and

$$
1-P(x)=(1-x) R(x) .
$$

Since $m<\infty$ the power series for $R(x)$ converges absolutely and uniformly in $|x| \leqq 1$. We claim that $R(x)$ has no zeros for $|x| \leqq 1$. For $|x|<1$ this is clear from (2), since $P(x)$ has positive coefficients

Received by the editors March 1, 1948.

1 To be published elsewhere. The theorem and method of the present paper were extended to the continuous case by D. Blackwell, $A$ renewal theorem, Duke Math. J. vol. 15 (1948) pp. 145-150. This research originated from work under an ONR contract at Cornell University. 
and therefore $|P(x)|<1$, for $|x|<1$. Then any zeros of $R(x)$ must occur on the circle $|x|=1$. From (1) it follows that $R(1)=m \neq 0$. Hence any zero must be of the form $x_{0}=e^{i \theta_{0}}, 0<\theta_{0}<2 \pi$. If $R\left(x_{0}\right)=0$, then (2) implies $P\left(e^{i \theta_{0}}\right)=1$. Since $p_{k} \geqq 0$ this can happen only if $\cos \theta_{0} k=1$ for all $k$ for which $p_{k} \neq 0$. But this is impossible because $P(x)$ would be a power series in $x^{t}$, for some integer $t>1$.

Then the function

$$
\frac{1-x}{1-P(x)}=\frac{1}{R(x)}
$$

has no singularities in $|x|<1$ and we can expand it in a power series

$$
\frac{1}{R(x)}=\sum_{n=0}^{\infty} a_{n} x^{n}
$$

where

$$
a_{n}=\frac{1}{2 \pi i} \int_{|x|=r} \frac{x^{-n-1}}{R(x)} d x
$$

Now $[R(x)]^{-1}$ is bounded in $|x|<1$; therefore we can apply Lebesgue's convergence theorem to let $r \rightarrow 1$; we obtain

$$
a_{n}=\frac{1}{2 \pi i} \int_{|x|=1} \frac{x^{-n-1}}{R(x)} d x=\frac{1}{2 \pi} \int_{-\pi}^{\pi} \frac{e^{-i n \theta}}{R\left(e^{i \theta}\right)} d \theta,
$$

for $n=0,1, \cdots$.

But we have already seen that

$$
R\left(e^{i \theta}\right)=\sum_{n=0}^{\infty} r_{n} e^{i \theta n}
$$

converges absolutely and has no zeros. It follows by a theorem of Wiener ${ }^{2}$ that $\left[R\left(e^{i \theta}\right)\right]^{-1}$ has an absolutely convergent expansion

$$
\frac{1}{R\left(e^{i \theta}\right)}=\sum_{n=-\infty}^{\infty} b_{n} e^{i n \theta}
$$

where $\sum\left|b_{n}\right|<\infty$ and

$$
b_{n}=\int_{-\pi}^{\pi} \frac{e^{-i n \theta}}{R\left(e^{i \theta}\right)} d \theta, \quad-\infty<n<\infty .
$$

A comparison of this and (4) shows that $\sum\left|a_{n}\right|<\infty$. From (3) we

2 A. Zygmund, Trigonometrical series, Warsaw, 1935, p. 140. 
can conclude that $\sum a_{n}=m^{-1}$. But

$$
\sum_{n=0}^{\infty} a_{n} x^{n}=(1-x) \sum_{k=0}^{\infty} u_{k} x^{k}
$$

It follows from Abel's theorem that $u_{k} \rightarrow 1 / m$.

This argument does not work in the case $m=\infty$ since then $R(x)$ is not bounded. The following proof is quite elementary and does not distinguish between the two cases.

Clearly

$$
u_{n}=p_{0} u_{n}+p_{1} u_{n-1}+\cdots+p_{n-1} u_{1}+p_{n} u_{0}, \quad n \geqq 1 .
$$

Moreover, since $R(x) U(x)=(1-x)^{-1}$, we have

$$
r_{0} u_{n}+r_{1} u_{n-1}+\cdots+r_{n-1} u_{1}+r_{n} u_{0}=1 \text {. }
$$

Let

$$
\lambda=\lim \sup u_{n},
$$

and let $\left\{n_{\nu}\right\}$ be a sequence such that $u_{n_{\nu}} \rightarrow \lambda$. We claim that for any fixed $j>0$ for which $p_{j}>0$ we have $u_{n_{\nu}-j} \rightarrow \lambda$. In fact, assume that $u_{n_{\nu}-j} \rightarrow \lambda^{\prime}<\lambda$. Then for sufficiently large $\nu$

$$
\begin{aligned}
\lambda-\epsilon & <u_{n_{\nu}} \\
& <\left(p_{0}+\cdots+p_{j-1}+p_{j+1}+\cdots+p_{N}\right)(\lambda+\epsilon)+p_{j} \lambda^{\prime}+\epsilon \\
& \leqq\left(1-p_{j}\right)(\lambda+\epsilon)+p_{j} \lambda^{\prime}+\epsilon<\lambda-p_{j}\left(\lambda-\lambda^{\prime}\right)+2 \epsilon
\end{aligned}
$$

whence $\lambda^{\prime}=\lambda$.

Repeating the same argument, we see that

$$
\lim _{\nu \rightarrow \infty} u_{n_{\nu}-8 j}=\lambda
$$

for every fixed integer $j \geqq 0$, provided only that $u_{n_{\nu}} \rightarrow \lambda$ and $p_{j}>0$. Now consider the set of all integers $j$ for which $p_{j}>0$. By hypothesis, their greatest common divisor is 1 . We can, therefore, find a finite collection $a_{1}, a_{2}, \cdots, a_{t}$ of subscripts such that $p_{a_{1}}>0, \cdots, p_{a_{t}}>0$ and that their greatest common divisor is 1 . Then by (5)

$$
\lim _{\nu \rightarrow \infty} u_{n_{\nu}-k}=\lambda
$$

for every fixed integer $k$ of the form

$$
k=x_{1} a_{1}+x_{2} a_{2}+\cdots+x_{t} a_{t} .
$$

However, every integer $k>a_{1} a_{2} \cdots a_{t}$ can be put into the form (7) 
and hence (6) holds for every sufficiently large fixed $k$. Now put in (5) $n=N_{\nu}=n_{\nu}-a_{1} a_{2} \cdots a_{t}$. Then for every fixed $M$

$$
1 \geqq r_{0} u_{N_{\nu}}+r_{1} u_{N_{\nu}-1}+\cdots+r_{M} u_{N_{\nu}-M} \text {. }
$$

As $\nu \rightarrow \infty$ all terms $u_{N_{\nu}-k} \rightarrow \lambda$ and hence

$$
1 \geqq \lambda\left(r_{0}+r_{1}+\cdots+r_{M}\right)
$$

or $\lambda \leqq 1 / m$ (with $\lambda=0$ if $\sum r_{n}=\infty$ ).

If $m<\infty$ we can use a similar argument for $\mu=\lim$ inf $u_{n}$ to show that $\mu \geqq 1 / m$. This proves the theorem.

SyRACUSE UNIVERSITY AND

CORNELl UNIVERSITY

\section{A CONSISTENCY THEOREM}

\section{H. D. BRUNK ${ }^{1}$}

1. Introduction. Of primary importance in a theory of representation of functions by series which do not necessarily converge is its consistency theorem, which states that if a series which represents a function $F$ converges to a function $\Phi$, then $F \equiv \Phi$. Such a theorem for asymptotic representation in a strip region of a function by Dirichlet series with a certain logarithmic precision, an idea introduced by Mandelbrojt [1], ${ }^{2}$ is the subject matter of this note. From it follow similar theorems for less general extensions of the idea of asymptotic series. The method consists in using the proof of the fundamental theorem in [1] to set up a homogeneous linear differential equation of infinite order with constant coefficients, which must be satisfied by the difference $F-\Phi$; then applying a method of Ritt to show that the only solution is identically zero.

The notation used by Mandelbrojt in [1] will be used here also. Let $\left\{\lambda_{n}\right\}$ be an increasing sequence of positive numbers $\left(0<\lambda_{n} \uparrow\right)$. Denote by $N(\lambda)$, defined for $\lambda>0$, the distribution function of $\left\{\lambda_{n}\right\}$; that is, the number of terms in the sequence $\left\{\lambda_{n}\right\}$ less than $\lambda$; and by $D(\lambda)$ the density function of $\left\{\lambda_{n}\right\}: D(\lambda)=N(\lambda) / \lambda$. Let $D^{\circ}$ represent the upper density: $D^{\circ}=\lim \sup _{\lambda \rightarrow \infty} D(\lambda)$; and $D^{\circ}(\lambda)$ the upper density function of $\left\{\lambda_{n}\right\}: D^{\cdot}(\lambda)=1$. u.b. $x \geqq \lambda D(x)$; clearly $D^{\cdot}(\lambda)$ is continuous and decreases to $D^{*}$ (unless $D^{\circ}(\lambda) \equiv D^{*}=\infty$ ).

Presented to the Society, April 17 1948; received by the editors March 15, 1948.

1 The author is indebted to Professor Mandelbrojt for suggesting the problem considered in this note.

${ }^{2}$ Numbers in brackets refer to the bibliography at the end of the paper. 